\title{
Uncovering the physics of flapping flat plates with artificial evolution
}

\author{
By MICHELE MILANO AND MORTEZA GHARIB \\ Graduate Aeronautics Laboratories, Option of Bioengineering, California Institute of Technology, \\ 1200 E. California Blvd, Pasadena, CA 91125, USA
}

(Received 29 March 2005 and in revised form 13 April 2005)

We consider an experiment in which a rectangular flat plate is flapped with two degrees of freedom, and a genetic algorithm tunes its trajectory parameters so as to achieve maximum average lift force, thus evolving a population of trajectories all yielding optimal lift forces. We cluster the converged population by defining a dynamical formation number for a flapping flat plate, thus showing that optimal unsteady force generation is linked to the formation of a leading-edge vortex with maximum circulation. Force and digital particle image velocimetry measurements confirm this result.

\section{Introduction}

There is a wealth of research work on unsteady force generation by flapping wings, especially related to insect flight. Since the pioneering work of Weis-Fogh (1973), researchers have addressed the problem with a variety of approaches. Ellington (1984) examined the idea that leading-edge vortices generated by insect flapping wings may be primarily responsible for lift generation; in later studies, Ellington et al. (1996) and Dickinson, Lehmann \& Sane (1999) visualized the three-dimensional structure of vorticity generated by an insect wing, by analysing wing models replicating actual flapping kinematics by insects. By using wing models, Birch \& Dickinson (2001) also analysed in more detail the behaviour of the leading-edge vortices produced by insect wings; in these studies, leading-edge vortices have been observed to be responsible for a significant part of the lift force produced by a flapping insect wing. Liu et al. (1998) and Ramamurti \& Sandberg (2002) did simulations using computational fluid dynamics, confirming the previous experimental results.

Here we try to better quantify the link between vorticity dynamics and force production for flapping wings. With this aim, we conducted a series of experiments in which a rectangular flat plate with aspect ratio 6 performs a flapping motion with two degrees of freedom. This geometry is a simplified model of an insect wing, and the motion profile models that of an insect wing during a stroke. We are interested in studying systematic parameter correlations that are then used to develop a physical model explaining maximum lift generation. We parameterize the plate trajectory by imposing a general periodic flapping motion, and then we search the resulting parameter space for solutions yielding an average lift force greater than a predefined threshold. To search the parameter space systematically we use a Genetic Algorithm (GA) that has been developed by Milano (2002) to generate a cluster of parameter combinations distributed in an optimum basin, thus allowing a better physical understanding of phenomena by studying the shape of the converged population. The GA has been successfully validated by Milano \& Koumoutsakos (2002) in the context of the study of fluid 


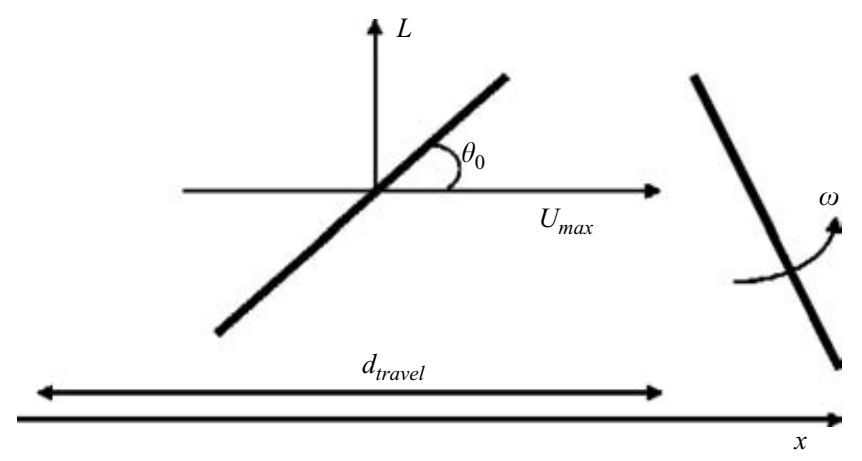

FIGURE 1. Sketch of the plate trajectory, where the plate is viewed from the top. See the main text for definition of the parameters.

dynamics problems, by studying the benchmark problem of drag reduction for a cylinder flow.

When applying the GA to our experiment, we found that the converged parameters did not present significant correlations; however, based on the kinematic trajectories that the solutions parameterize, we defined a dynamic parameter that quantifies the formation of the leading-edge vortices produced by the flapping plate. This dynamic formation parameter is a generalization of the one defined by Gharib, Rambod \& Shariff (1998), obtained in the same spirit as the generalization obtained for jets with varying exit nozzle by Dabiri \& Gharib (2005). By force measurements and digital particle image velocimetry (DPIV) measurements of the startup vortices produced by flat plates following an optimal trajectory, we show that the dynamical formation parameter properly identifies trajectories that produce vortices of maximum circulation, thereby maximizing the average lift produced during periodic flapping.

\section{Problem definition and experimental setup}

We focus on the problem of maximizing the average lift produced by a flat plate flapping with two degrees of freedom, i.e. rotation and translation. Figure 1 sketches the trajectory followed by the plate, showing also the parameters defining it; the $x$ position of the plate is the position of its centre chord in the plane of the trajectory, and the translational direction is considered positive when the plate translates to the right. The plate travels a distance $d_{\text {travel }}$ with a constant velocity $U_{\max }$ and constant angle of attack $\theta_{0}$, then it inverts its motion from a positive to negative direction by rotating with constant angular velocity $\omega$ and changing its translational velocity as follows:

$$
\left.\begin{array}{l}
u(t)=U_{\max } \frac{\cos (\omega t)}{\cos \left(\theta_{0}\right)}, \\
t \in\left[\frac{\theta_{0}}{\omega}, \frac{2 \theta_{0}+\pi}{2 \omega}\right],
\end{array}\right\}
$$

where the initial time is taken at the beginning of the turn; the turn from a negative to positive direction is symmetrical. The plate trajectory is periodic because the angular velocity during a turn is defined to be equal to the frequency $\omega$ of the translational part of the turn, in (2.1). This is the only constraint imposed on the plate trajectory, besides limits for $U_{\max }$, that varied between 0 and $15 \mathrm{~cm} \mathrm{~s}^{-1}$, for $\omega$, that varied between 0.1 and $30 \mathrm{rads}^{-1}$, and for $d_{\text {travel }}$, that was allowed to vary between 0 and 

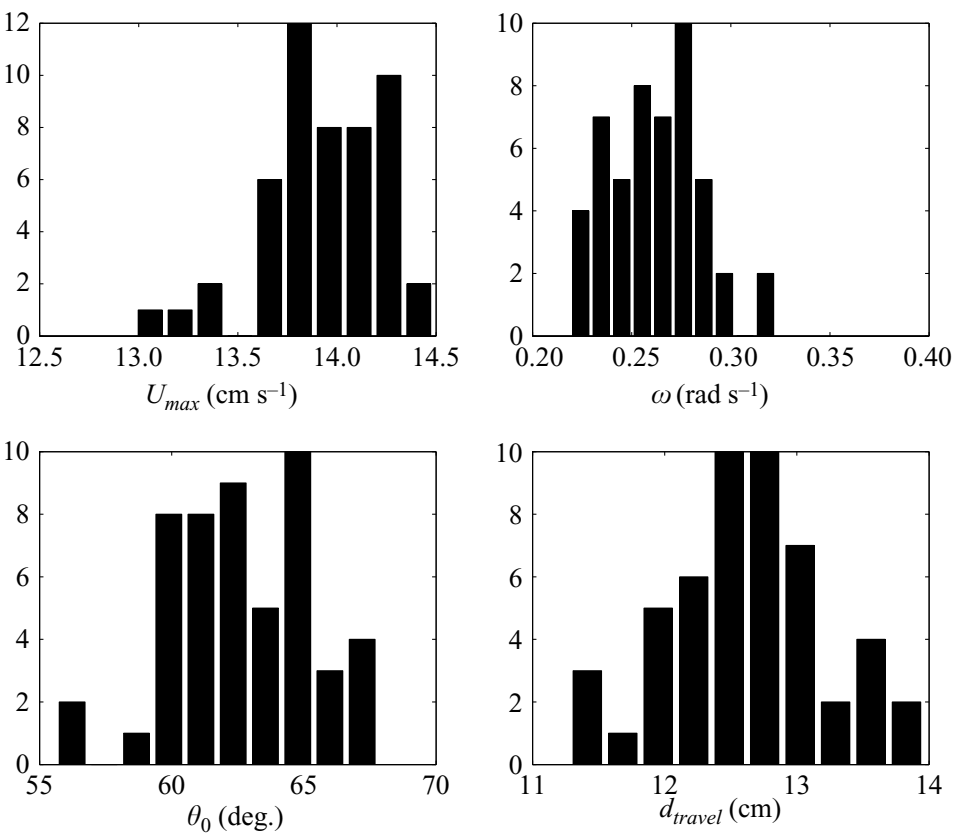

FIGURE 2. Histogram of the converged GA population. Analysis of covariance matrix revealed no linear correlations between parameters.

$20 \mathrm{~cm}$. Experiments were done in a water towing tank, in which an aspect ratio 6 flat plate was vertically mounted on a force balance measuring the lift force. The plate chord length $c=5 \mathrm{~cm}$, and the minimum distance of the tank walls from the plate edges was 4 chord lengths, thus making wall effects negligible. Two computer-controlled stepper motors provided the desired trajectories, one controlling the rotational motion and another one controlling the translational motion of the plate. For each experiment the plate was flapped four consecutive times, and the lift $l(t)$ was measured and integrated over time; the function that we maximized was

$$
L=\int_{0}^{4 T} l(\tau) \mathrm{d} \tau
$$

where $T$ is the stroke period. In order to avoid disturbances from previous runs, there was a pause of two minutes at the end of each experiment to allow the water to settle.

\section{Results from the genetic algorithm}

The GA used to tune the trajectory parameters is a clustering GA developed by Milano (2002); it is specifically designed to cluster a set of parameters (population) inside optimum basins, thus allowing analysis of parameter correlations. Each element of the GA population is a vector $\boldsymbol{x}_{\mathrm{i}}=\left\{U_{\text {max }}, d_{\text {travel }}, \theta_{0}, \omega\right\}_{\mathrm{i}}$, comprising four components, which encode a particular trajectory followed by the plate as specified in (2.1). The GA evolved this population so that its elements were distributed inside an optimum basin. We used a population of 50 elements, which in the four-dimensional parameter space considered converged in about 3000 iterations. The quantity that we maximized was the average lift over the first 4 strokes of the plate, as detailed before. The converged population is represented as a set of histograms in figure 2 . 

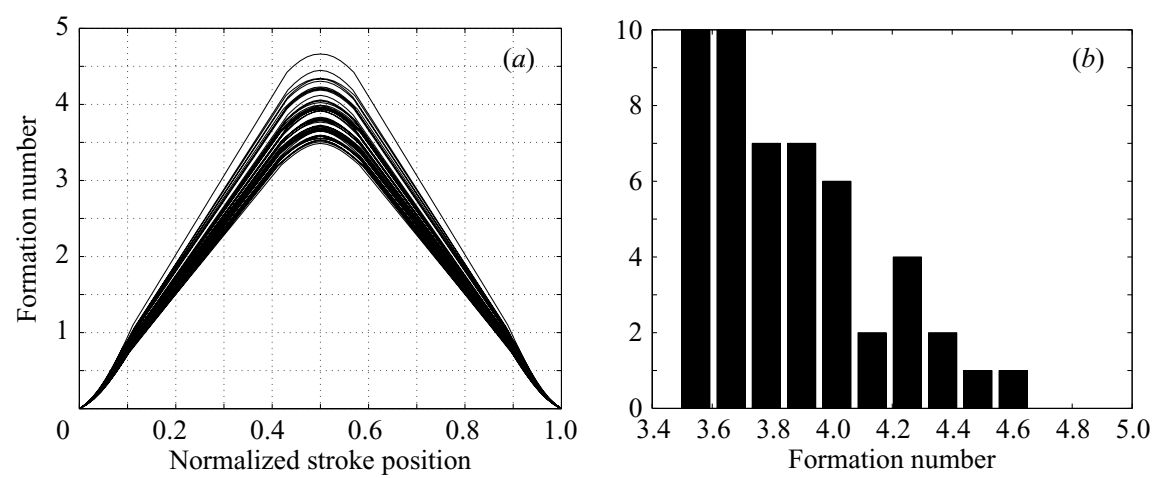

FIGURE 3. (a) Formation parameter of optimal solutions as a function of normalized stroke length; $(b)$ histogram of the peak formation parameter computed for the optimal set. A marked clustering of the peaks between 3.6 and 4.6 is observed, an indication that optimal solutions produce vortices of maximum circulation at each half-stroke.

The Reynolds numbers of the converged solutions are of the order of 6000 , based on the plate chord length and maximum velocity. This order of magnitude is within the range of typical Reynolds numbers for larger insects (Weis-Fogh 1973).

By analysing the covariance matrix of the population parameters, we found no significant linear correlations between parameters yielding optimal average lift.

\section{Clustering of the results with a dynamic formation parameter}

Having generated a set of equally optimal solutions for the problem, we proceeded to study this set in order to uncover common features. Since the covariance matrix revealed no significant linear correlations between parameters, we now seek other kinds of correlations and common features, by comparing the length scales for leading-edge vortex formation for the set of solutions. This is done by introducing a dynamical formation parameter for a flat plate undergoing an arbitrary twodimensional trajectory. We extend the definition of formation parameter given by Gharib et al. (1998) in the same spirit as in the work by Dabiri \& Gharib (2005), that defined a dynamic formation parameter and used it to characterize vortical structures produced by jets with variable exit diameter geometry. We define a dynamical formation time for a flat plate undergoing a two-dimensional trajectory as follows:

$$
T^{*}(t)=\int_{t_{0}}^{t} \frac{u(\tau)+\frac{1}{2} c \omega(\tau) \cos (\theta(\tau))}{c \sin (\theta(\tau))} \mathrm{d} \tau
$$

where $u$ is the plate translational velocity, $c$ is the plate chord length, $\omega(t)$ is the instantaneous angular velocity, and $\theta(t)$ is the instantaneous angle of attack. The quantity being integrated is the ratio of the horizontal distance travelled by the plate leading edge and the projected chord length; in this way the plate rotation is properly taken into account when determining the stage of the leading-edge vortex formation. Figure 3 reports formation times for the entire population, as a function of normalized stroke length, and a histogram of the peak formation times; all the peak formation times cluster in the region 3.6-4.6. This order of magnitude for the peak dynamic formation time is typical of systems producing vortices of maximum possible circulation as showed by Gharib et al. (1998) and Dabiri \& Gharib (2005); therefore if the formation time as defined by (4.1) can be shown to be 


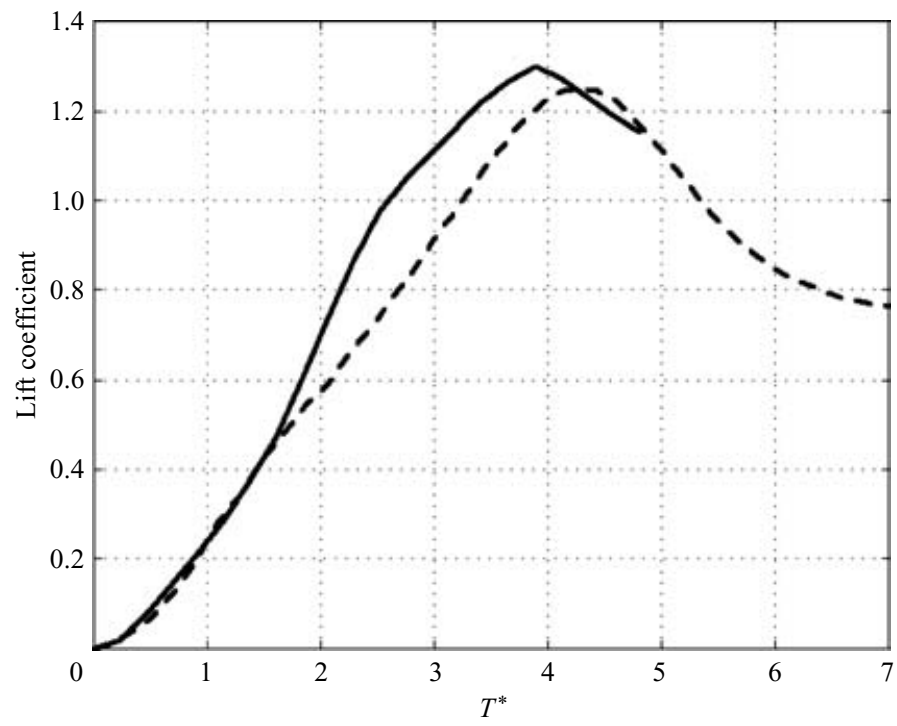

FigURE 4. Lift coefficient produced by half a stroke of one of the optimal solutions (continuous line), compared with the lift coefficient produced by the same flat plate, but translating with a constant velocity and angle of attack (dashed line). The continuous line stops when the plate is about to change its direction, for the optimal solution.

related to the formation of maximum-circulation vortices, then this result is a clear indication that optimally efficient solutions tend to generate leading-edge vortices of maximum strength. Thus, as a further validation, we considered one of the solutions $\left(U_{\max }=14 \mathrm{~cm} \mathrm{~s}^{-1}, d_{\text {travel }}=12.5 \mathrm{~cm}, \theta_{0}=62^{\circ}, \omega=0.25 \mathrm{rad} \mathrm{s}^{-1}\right)$ and we compared lift coefficient and vorticity produced by the first half-stroke of the plate, with the same quantities as measured in the case of a plate that undergoes a purely translational motion, with a constant angle of attack $\theta_{0}$ and constant velocity $U_{\max }$. Figure 4 compares the lift forces for the two cases, showing that the optimal half-stroke is able to produce a better transient lift force. Figure 5 compares vorticity of DPIV measurements taken at a $50 \%$ spanwise location for the two cases, showing that for the plate undergoing pure translation the pinch-off process of the leading-edge vortex starts immediately after the end of the half-stroke for the plate following the optimal trajectory.

To assess when saturation of the leading-edge vortex occurs during a half-stroke for an optimal and suboptimal case, figure 6 compares circulation of the leadingedge vortex for the same solution mentioned before and for a different trajectory, obtained by making the total distance travelled during a half-stroke $20 \%$ longer. The circulation of the leading-edge vortex saturates before the end of the half-stroke for the suboptimal solution, whereas for the optimal solution saturation occurs near the end of the half-stroke.

Thus the optimal half-stroke produces a vortex of maximum circulation, and this is indicated by a formation time of about 4, consistently with the results obtained for jet flows by Gharib et al. (1998) and Dabiri \& Gharib (2005). Based on this result, we can call the peak formation time during an optimal stroke the 'formation number', denoting the formation time at which a leading-edge vortex of maximum circulation is produced. 


$$
T^{*}=3.5
$$
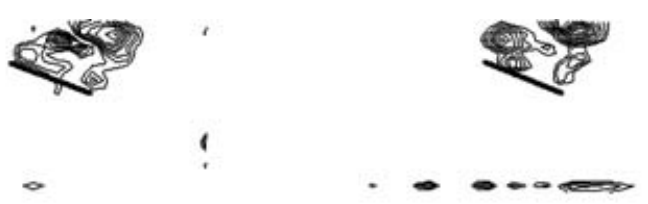

$$
T^{*}=4
$$
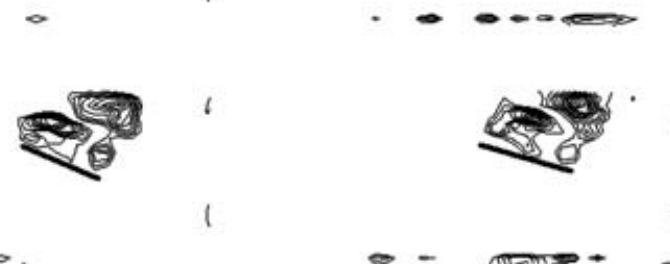

$$
T^{*}=4.8
$$
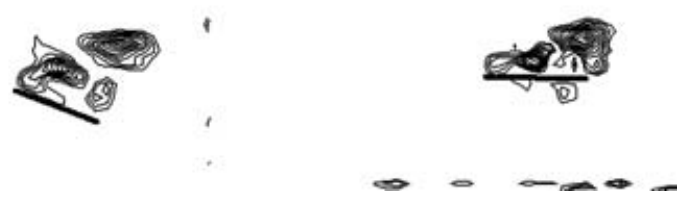

Figure 5. Comparison between vorticity contours of DPIV measurements for a flat plate translating with constant velocity and angle of attack (left column) and a flat plate performing the first half-stroke of an optimal trajectory profile. In both cases the plate moves towards the bottom of the figure, so the vortical structures on the right-hand side of the plates are the leading-edge vortices, whereas the vortices on the left-hand side are trailing-edge vortices. In the optimal profile, the plate starts turning back (counterclockwise) just before the leading-edge vortex pinch-off, thus avoiding it.

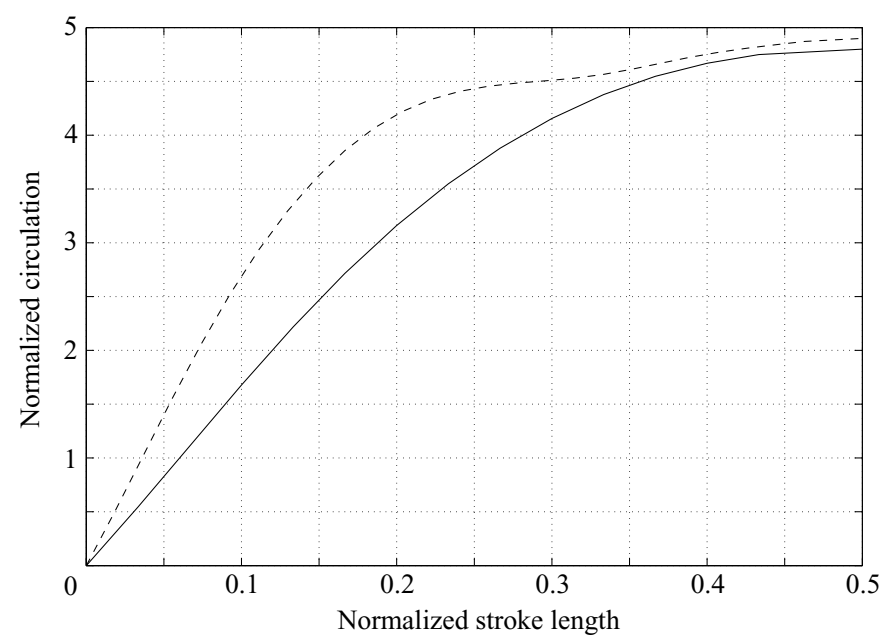

FIGURE 6. Normalized circulation of the leading-edge vortex estimated from DPIV measurements, for an optimal half-stroke (continuous line) and a suboptimal one which is $20 \%$ longer (dashed line). Abscissas are normalized with the length of the full stroke.

\section{Conclusions}

By applying a clustering GA to the problem of lift maximization for a flapping flat plate, we were able to generate a set of optimal solutions, i.e. trajectories that yield maximum average lift. We studied the converged population in order to uncover common features, and we found that optimal flapping produces leading-edge vortices of maximum circulation; we introduced a dynamic formation time that captures this 
common feature for the population, and we found that a dynamic formation time of about 4 is associated with production of a maximum-circulation vortex, consistently with past experimental results obtained for jet flows by Gharib et al. (1998) and Dabiri \& Gharib (2005). Future work will involve extension of these results to more complex shapes and trajectories, and an assessment of three-dimensional effects induced by the plate tip.

\section{REFERENCES}

Birch, J. M. \& Dickinson, M. H. 2001 Spanwise flow and the attachment of the leading-edge vortex on insect wings. Nature 412, 729-733.

DABIRI J. O. \& GHARIB, M. 2005 Starting flow through nozzles with temporally variable exit diameter. J. Fluid Mech. to appear.

Dickinson, M. H., Lehmann, F. O. \& Sane, S. P. 1999 Wing rotation and the aerodynamic basis of insect flight. Science 284, 1954-1960.

Ellington, C. P. 1984 The aerodynamics of hovering insect flight. Phil. Trans. R. Soc. Lond. B 305, $1-181$.

Ellington, C. P., Van den Berg C., Willmott A. P. \& Thomas, A. L. R. 1996 Leading-edge vortices in insect flight. Nature 384, 626-630.

Gharib, M., Rambod, E. \& Shariff, K. 1998 A universal time scale for vortex ring formation. J. Fluid Mech. 360, 121-140.

Liu, H., Ellington, C. P., Kawachi, K., Van den Berg, C. \& Willmott, A. P. A. 1998 Computational fluid dynamic study of hawkmoth hovering. J. Exp. Biol. 201, 461-477.

Milano, M. 2002 Machine learning algorithms for flow modeling and control. PhD thesis 14523, Swiss Federal Institute of Technology (ETH).

Milano, M. \& Koumoutsakos, P. 2002 A Clustering Genetic Algorithm for cylinder drag optimization J. Comput. Phys. 175, 79-107.

Ramamurti, R. \& Sandberg, W. C. 2002 A three-dimensional computational study of the aerodynamic mechanisms of insect flight. J. Expl Biol. 205, 1507-1518.

WeIS-FoGH, T. 1973 Quick estimates of flight fitness in hovering animals, including novel mechanisms for lift production. J. Expl Biol. 59, 169-230.

Willert, C. \& Gharib, M. 1991 Digital Particle Image Velocimetry. Exps. Fluids 10, 181-193. 